\title{
The importance of task appropriateness in computer-supported collaborative learning
}

\author{
Kathy Buckner and Kate Morss \\ Queen Margaret College, email:kbuckner@mail.qmced.ac.uk
}

Collaborative learning activities can be beneficial for exchanging ideas, sharing experiences, and developing shared understanding. It is our view that the task given to the student is central to the success or otherwise of the learning experience. In this paper, we discuss the need for the adaptation of traditional face-to-face tasks when these are incorporated in computer-supported collaborative learning (CSCL) environments. We focus on critical issues in relation to the implementation of CSCL tasks including: the appropriateness of the medium for the task, the role of individuals, the volume of work involved, the time allocated for tasks or sub-tasks, and, the assessment procedures.

In this paper we describe and evaluate two case studies that illustrate the importance of the appropriateness of the task in computer-supported co-operative learning. Both case studies (taught by one of the authors) involve final-year Information Management undergraduates in on-campus modules. Our findings indicate that the task must allow the module outcomes to be achieved (and assessed if necessary), and must be supported by software tools which facilitate effective student learning. If it does, and the assessment mechanism is appropriate, the benefits include improved motivation to participate in discussion, improved student learning, and the ability to assess individual performance in group activities.

\section{Introduction}

The study of learning in collaborative electronic environments is becoming established as Computer Supported Collaborative Learning (CSCL) - an emergent sub-discipline of the more established Computer Supported Co-operative Work (CSCW) discipline (Webb, 1995). Using computers for the development of shared understanding through collaboration has been explored by Crook who suggests that success may depend partly on having a clearly specified purpose or goal (Crook, 1994). It is our view that the appropriateness of 
the task given to the student is central to the success or otherwise of the learning experience. However, the tasks that are given to facilitate collaborative learning in face-toface situations are not always suitable for direct transfer to the electronic medium. It may be necessary to consider redesigning these tasks in relation to the medium in which they are to be undertaken and the functionality of the electronic conferencing software used.

The way in which CSCL tasks are implemented in terms of assessment of learning outcomes (allocation of marks to individuals and/or to the entire group), and group process and operation (e.g. anonymity, role of individual, role of tutor) is also significant. The enforced requirement (or otherwise) to participate in assessed or non-assessed activities is likely to affect the amount and quality of contribution in any CSCL activity (McAteer et al., 1997). Slavin (1996) examines in depth the theoretical perspectives which could help to explain student achievement in co-operative learning (not necessarily CSCL) situations. He suggests that factors affecting achievement include group and individual accountability, social and group cohesion, and the ability to learn directly from peers and indirectly from supporting and instructing peers. These are issues that we consider in the work presented here. In this paper we describe and evaluate two campus-based case studies that illustrate the importance of task appropriateness in computer-supported collaborative learning.

\section{The case studies}

\section{Case Study I: Computer-assisted learning: electronic seminar presentation}

The first case study is one element of a final-year optional module in computer-assisted learning provided on the BA (Hons) Information Management course at Queen Margaret College. As part of the assessment, students used COW software (Conferencing on the Web, http://thecity.sfsu.edu/COW2/, an asynchronous computer conferencing program, hosted by NetLinkS http://netways.shef.ac.uk/ at the University of Sheffield) to deliver an electronic seminar presentation, lead and finally summarize the ensuing discussion of the topic. Students were graded according to their ability both to lead and to contribute to the debate. Each student was asked to select a topic for their seminar presentation and two or three students (in a class of 13) were assigned as conference leaders to each of six topics. In their role as conference leaders students were required to make an individual opening statement of 500 words incorporating appropriate references from print and electronic sources by a specific date. Contributors had one week to make two contributions to each of the conferences they were assigned. They were required to take into account the originator's opening statement and contributions from other participants, incorporate additional evidence from print and/or electronic sources, and reflect on their own experience. The discussion was followed by a 500 -word closing statement by each conference leader. The conference leaders were expected to be proactive in stimulating discussion. Each student was assigned by the lecturer as a contributor to two additional conferences and was required to participate in one further conference of their own choice. This element of the module accounted for 33 per cent of the marks. No group marks were awarded for this activity.

\section{Case Study 2: Human factors for information managers: problem-solving}

The second case study is one element of an undergraduate final-year compulsory module in Human Factors for Information Managers which operates within a networked learning 
environment (NLE) (Buckner and Davenport, 1996). The collaborative aspect discussed here involved the students in a problem-solving activity that used Netscape Navigator News as a platform for asynchronous discussion and electronic submission of an agreed solution. Each group of four students (in a class of 24) was allocated to a specific problem which was accessible via the NLE. Much of the information required to solve the problem was available within the NLE, although traditional print material and other online resources were also utilized. Students were informed that all discussion and debate should take place online in the newsgroup and that this electronic record would form the basis for the individual and group assessment. Additionally they were allowed to use email to pass documents between each other provided that they copied any such transactions to the tutors. It was felt that this might be an easier mode of working as they neared a final outcome for the group solution to the problem. Students were advised to work together as a team to solve the problem and it was suggested that they might want to assign different roles to specific individuals, e.g. moderator/team leader, or to take responsibility for gathering information on specific topics. The final solution was to be no more than 500 words long. A total of 30 per cent of the marks available for this module was allocated to the problem-solving activity, 15 per cent for the individual contribution and 15 per cent for the group solution to the problem.

\section{Evaluation of the case studies}

A multi-instrument evaluation methodology was adopted for both case studies (Draper et al., 1994). Questionnaires with a mix of open-ended and attitudinal questions were used to obtain feedback about the tasks undertaken, the appropriateness of the software provided for performing the task and operational issues. There were nine respondents from Case Study 1 (69 per cent), 18 respondents from Case Study 2 (75 per cent). A focus group discussion (involving three students who had participated in both case studies) was undertaken by an independent researcher to enable the issues to be explored in more depth. In addition, the students' assessments (electronic discussion, electronic conference presentations and debates) were examined.

\section{Analysis and discussion}

In both case studies the students were quite clear about the nature of the exercise (the task). Their roles within the conferences and newsgroup were understood, although these were more clearly defined by the tutor in the seminar presentation case study.

In Case Study 1 (Seminar Presentation) more than three-quarters of the students thought that they were better informed both as a presenter (or seminar leader) and as a contributor than in face-to-face discussions. There was less tutor intervention than in face-to-face seminars where student contributors are often reluctant to take an active part in discussion about another student's topic. The tutor and students thought that contributions to discussion were of a higher standard than in traditional seminars. Students felt that: i) it was easier to recap on earlier parts of the discussion, ii) they were able to follow up, in an informed way, on responses from other students, and iii) the medium encouraged more open participation (particularly by the more reserved students). 
Referring to traditional seminars one student stated:

... in the classroom situation ... maybe two or three people speak all the time and people tend to sit back and not bother to say anything.

A second student agreed:

$\ldots$ or if somebody is actually saying what you are thinking there is no point . . . you coming out and saying it as well.

In Case Study 2 (Problem-solving), students felt that using newsgroups was beneficial for sharing research findings but made it harder to determine a final solution to the problem than if they had been using face-to-face communication. There was a feeling that they were 'waiting' for responses, that it was more time-consuming and less 'instant' than face-to-face debate. Some groups (with the approval of the lecturer) resorted to meeting face-to-face to produce the final submission document.

The quality and quantity of contributions was affected by two main factors: assessment and motivation. In both case studies the students' ongoing contribution to the discussion was assessed as well as the opening statement (in Case Study 1) and the final submission. Because contributions were to be inciuded in the assessment each posting was identified as being the work of a particular student. For Case Study 2, students appreciated being able to obtain an individual as well as a group mark. They found the transparency which the software provided (identifying them as authors of their contributions) was valuable in this respect as they could be confident that they would get credit for their own effort.

Whilst it was apparent that a few students contributed to the debate only because they were being assessed, others contributed over and above the required number of times. Motivational factors, such as personal interest in the subject matter, pride in their contribution to the debate, the ability of the seminar leaders to generate discussion (by asking thought-provoking, challenging or probing questions) and feedback from their peers, were influential in this respect and also affected the quality of the submissions.

In both case studies there were specific benefits in terms of learning. Students learned from each other, were motivated because input from other students prompted further research, and spent considerable effort in finding evidence (both print and Web-based) to support their arguments.

Almost all the students enjoyed having the freedom to decide when to contribute to the debates, although there was less agreement on whether it was easy or difficult to organize their time for regular interaction. Those who had access to a computer and modem at home (67 per cent in Case Study 1, 49 per cent in Case Study 2) enjoyed increased flexibility over choice of time and place to participate in the discussion, whilst students working from college also felt there was a benefit in not being constrained by a specific two-hour timetabled class.

For example, one student stated:

It suits me as a Mum. I could put more in and research in my own time and then come in and put in my information when I'm free.

Furthermore, they felt that the length of time allotted to the debates (two/three weeks overall) led to the development of a more in-depth understanding of the topics. 


\section{Conclusions}

There are several issues to be considered when defining and setting up tasks for computersupported collaborative learning, including the appropriateness of the medium for the task, the role of individuals, the volume of work, the time allocated for tasks or sub-tasks, and the assessment procedures.

In the case studies outlined here it is our view that the task defined in Case Study 1 was entirely appropriate for the medium (a similar task utilizing different software is described by McAteer et al. (1997)). Students were clear about the nature of the task, their role (see Berge (1995) for a description of the role of facilitators in computer conferences) and the procedures to be followed. Some criticism was made of the fact that COW did not allow for threading of messages, but this was a minor difficulty and on the whole the students found the interface easy to use. In terms of the task's comparability with traditional seminar presentations, greater emphasis was placed on the need for students to act as contributors to the debate (with this being assessed), although there was no verbal delivery of the paper. Even when the task is appropriate for the medium, it is important that implementers get the expected workload right. In this example students were critical of the number of conferences to which they had to contribute (four, including the one they led). Whilst this approach exposed them to a broad range of topics it also made it difficult for them to study any of the topics in sufficient depth. This will be reconsidered in future courses.

Whilst Case Study 1 provided a satisfactory outcome, Case Study 2 was on the whole less successful. Although the nature of the task was clear, it was less appropriate for the software environment used. The use of newsgroups was satisfactory for facilitating the sharing of research findings, but the task required a consensual decision and the mechanism provided for sharing and agreeing the content of the final submission was rather cumbersome (a different piece of software might well provide the necessary tools). If this exercise is run again it will be necessary to revise the task, rework the assessment details (the length of the final submission was rather short for a group exercise) and reconsider the appropriateness of the software environment.

To summarize:

- The task set in a CSCL environment must be appropriate for the medium. Discussion and debate is appropriate: attempting to arrive at a consensual view may not be, unless appropriate support tools can be provided.

- Electronic conferencing systems provide an excellent mechanism for recording, monitoring and assessing individual contributions to group activities.

- Students have time to reflect on contributions to the debate, undertake further research and make further contributions, leading to improved quality in student learning.

- The transparency of electronic discussion encourages participants to research topics thoroughly.

If the task and assessment are appropriate, and software provides the right support tools, then the quality of the learning and the learning experience will be improved. 


\section{References}

Berge, Z. L. (1995), 'Facilitating computer conferencing: recommendations from the field', Educational Technology, 35 (1).

Buckner, K. and Davenport, E. (1996), 'Support issues for case-based learning in an undergraduate human factors class', Education for Information, 14 (4), 331-42.

Crook, C. (1994), Computers and the Collaborative Experience of Learning, London: Routledge.

Draper, S. W., Brown, M. I., Edgerton, E., Henderson, F. P., McAteer, E., Smith, E. D., Watt, H. D., (1994), Observing and Measuring the Performance of Educational Technology, Teaching and Learning Technology Project - TILT, University of Glasgow.

McÁteer, E., Tolmie, A., Duffy, C. and Corbett, J. (1997), 'Computer-mediated communication as a learning resource', Journal of Computer Assisted Learning, 13 (4), 219-27.

Slavin, R. E. (1996), 'Research on cooperative learning and achievement: what we know, what we need to know', Contemporary Educational Psychology, 21 (1), 43-69.

Webb, B. R., 1995, 'Opinion: educational research and computer supported co-operative learning', Innovations in Education and Training International, 32 (2), 139-46. 